\title{
Efeitos de um jogo de tabuleiro sobre a seleção e consumo de alimentos por crianças
}

\author{
Effects of a board game on children's food selection and intake
}

\section{Efectos de un juego de mesa sobre la selección e ingesta de alimentos de los niños}

\author{
Mariana Gomide Panosso ${ }^{1}$ \\ Universidade Federal de São Carlos \\ Gabriele Gris \\ Universidade Federal de São Carlos \\ Silvia Regina de Souza \\ Universidade Estadual de Londrina
}

\begin{abstract}
Resumo
Objetivou-se avaliar os efeitos de um jogo de tabuleiro sobre as respostas de seleção e consumo de alimentos de diferentes grupos alimentares. Participaram três crianças (P1, P2 e P3) com idades entre cinco e seis anos. A pesquisa foi composta de três fases: linha de base (LB), intervenção/jogo e pósintervenção (PI). Na LB registrou-se a seleção de alimentos recorrendo-se a figuras e, posteriormente, o consumo desses alimentos no almoço. Na intervenção foram realizadas 12, 11 e 13 partidas com o P1, o P2 e o P3, respectivamente, nas quais competiram a pesquisadora e o participante. A pós-intervenção assemelhou-se à LB. Comparando-se os dados das três fases do P1 e P3 e da LB e PI do P2 constata-se que eles consumiram alimentos que não tinham por hábito consumir. Discutem-se tanto as variáveis que contribuíram para o aumento da variedade de seleção e consumo de alimentos como as limitações do estudo.

Palavras-chave: comportamento alimentar, educação alimentar infantil, jogo educativo
\end{abstract}

\begin{abstract}
The objective of this study was to evaluate the effects of a Board Game on the selection and consumption responses of foods from different food groups. Three children (P1, P2 and P3) aged between five and six years old participated. The research was composed of three phases: Baseline (LB), Intervention/play and Post-Intervention (IP). In LB, food selection using figures and the subsequent consumption of these foods at lunch was recorded. In the Intervention, 12, 11, and 13 games matches were played by P1, P2, and P3, respectively, between the researcher and the participant. The Post-Intervention resembled LB. Comparing the data from the three phases to P1 and P3, from LB and PI to P2, it is noted that they consumed foods that they did not use to. The procedural variables of the game that helped the participants to increase the variety of food selection and consumption, as well as study limitations, were discussed.

Keywords: eating behavior, child food education, educational game
\end{abstract}

\section{Resumen}

Se objetivó evaluar los efectos de un juego de mesa sobre las respuestas de selección y consumo de alimentos de diferentes grupos alimenticios. Participaron tres niños (P1, P2 y P3) con edades entre cinco y seis años. La investigación fue compuesta de tres fases: línea de base (LB), intervención / juego y post-intervención (PI). En la LB se registró la selección de alimentos por medio de figuras y el posterior consumo de estos alimentos en el almuerzo. En la intervención se realizaron 12, 11 y 13 partidos con el P1, P2 y P3, respectivamente, en los cuales compitieron la investigadora y el participante. La postintervención se asemejó a la LB. Comparando los datos de las tres fases para el P1 y P3 y de la LB y PI del P2 se constata que ellos consumieron alimentos que no tenían el hábito de consumir. Se discuten tanto las variables que contribuyeron para el aumento de la variedad de selección y consumo de alimentos así como las limitaciones del estudio.

Palabras clave: conducta alimentaria, educación alimentaria infantil, juego educativo

\footnotetext{
${ }^{1}$ Endereço de contato: Rua Piauí, 797, apto. 701 - CEP 86020-390- Londrina, PR.

E-mail: mariana_panosso@hotmail.com
} 
O comportamento alimentar é fundamental para a prevenção e o tratamento de doenças, uma vez que o excesso ou a carência de determinados nutrientes pode gerar problemas de saúde. De acordo com a World Health Organization (WHO, 2017), uma dieta pouco saudável é um dos principais fatores de risco para uma série de doenças crônicas, como as doenças cardiovasculares, o câncer, o diabetes, a obesidade etc. Recomendações gerais para uma dieta saudável são: comer mais frutas, legumes, leguminosas, nozes e grãos, e reduzir o sal, o açúcar e as gorduras. Todavia, muitas vezes, as escolhas alimentares são, na infância, muito seletivas, com quantidades elevadas de carboidrato, açúcar, gordura e sal e baixo consumo de alimentos como vegetais e frutas, se comparadas às quantidades recomendadas (Lowe \& Horne, 2009).

É na idade pré-escolar e no início da idade escolar que se formam os hábitos alimentares que acompanham o indivíduo ao longo da vida. Esses hábitos são fortemente influenciados por fatores genéticos e ambientais (Brasil, Devncenzi, \& Ribeiro, 2007). A espécie humana possui predisposição genética para rejeitar os sabores amargo e azedo e a responder preferencialmente aos sabores doces e salgados, além de preferir alimentos com alta densidade energética. A experiência do ser humano com os sabores começa durante o período de amamentação, como consequência do consumo alimentar da mãe (Birch, 1999). Com a introdução da alimentação sólida, o comportamento alimentar é aprendido no ambiente familiar e, secundariamente, por outras interações psicossociais e influências culturais (Oliveira, Araújo, Soares, \& Coimbra, 2016; Ramos \& Stein, 2000). Nesse processo de aprendizagem, muitas variáveis podem colaborar na escolha alimentar. Entre essas variáveis citam-se propagandas e comerciais de televisão (Costa, 2016), a modelação de pais, pares e irmãos (Birch, 1999; Rossi, Moreira, \& Rauen, 2008), consequências fisiológicas positivas ou negativas pós-ingestão (Birch, 1999), estratégias adotadas pelos pais para o consumo de determinados alimentos, status socioeconômico da família (Birch, 1999; Teixeira \& Moreira, 2016), fatores culturais (Wardle \& Cook, 2008) e disponibilidade e acesso facilitado aos alimentos (Birch, 1992).

A seletividade para o consumo alimentar realizada por muitas crianças pode ser resultado de práticas alimentares conduzidas de forma inadequada, embora crianças em fase de formação de hábitos alimentares, muitas vezes, não aceitam experimentar prontamente novos alimentos. Na fase pré-escolar e início da fase escolar, a velocidade do crescimento declina, e uma das consequências é a redução do apetite e da ingestão alimentar. Para que a rejeição a novos alimentos se modifique é necessário que a criança experimente os alimentos várias vezes, sem qualquer coerção (Birch, McPhee, Shoba, Pirok, \& Steinberg, 1987; Brasil et al., 2007).

Nos últimos anos, a partir dos estudos de Sidman (Sidman, 1971; Sidman \& Tailby, 1982 entre outros), pesquisas empregaram o modelo de equivalência de estímulos para investigar comportamentos comuns no contexto clínico, entre os quais o comportamento alimentar (e.g., Cardoso, 2007; Santos, 2017; Smeets \&Barnes-Holmes, 2003; Straatmann, Almeida, \& de Rose, 2014; Viana, 2010). Sidman e Tailby (1982) definem equivalência de estímulos como relações de estímulos condicionais derivadas que mostram as propriedades de reflexividade, simetria e transitividade. A propriedade de reflexividade implica a relação de igualdade que os estímulos de uma classe têm entre si (e.g., A1A1, B1B1 e C1C1); a simetria refere-se à reversibilidade funcional da discriminação condicional (i.e., se foi ensinado que "dado A1, 
escolher B1", então a relação "dado B1, escolher A1" deve emergir). Finalmente, na transitividade são necessários treinos anteriores: "dado A1, escolher B1" e "dado A1, escolher C1". Se o indivíduo, "dado B1, escolher C1" sem treino adicional, a propriedade de transitividade é demonstrada. Uma das vantagens da utilização do modelo de relações de equivalência de estímulos é que a formação de classes de estímulos equivalentes permite a eficiência no processo de ensino e aprendizagem, visto que o ensino de algumas relações pode produzir a emergência de outras que não foram diretamente ensinadas (Sidman, 1982). Ainda, símbolos abstratos podem adquirir valor positivo ou negativo, em razão de pertencerem a uma classe que contenha algum estímulo com significado, e influenciar escolhas e preferências por determinados produtos (Santos, 2017).

Em vista das vantagens do emprego desse modelo para o ensino de comportamentos específicos e da possibilidade do efeito de uma variável se estender aos demais membros de uma classe de equivalência, pesquisas interessadas no comportamento de escolhas alimentares têm sido desenvolvidas (Cardoso, 2007; Smeets \& Barnes-Holmes, 2003; Straatmann, 2008; Viana, 2006). Nessas pesquisas, bebidas (Smeets \& Barnes-Holmes, 2003), nomes impressos de alimentos (Straatmann et al., 2014) ou fotos de alimentos (Cardoso, 2007; Viana, 2006) foram relacionados, direta ou indiretamente a adjetivos impressos de alta e baixa palatabilidade (Cardoso, 2007; Viana, 2006), a fotos (Smeets \& Barnes-Holmes, 2003) ou a expressões faciais (Straatmann et al., 2014) que representavam felicidade ou tristeza (por exemplo, no trabalho de Smeets e Barnes-Holmes, houve formação de classes que continham figuras com significado positivo e negativo e símbolos abstratos que eram colocados em garrafas de refrigerantes, os quais controlaram as respostas dos participantes).

De modo geral, os dados obtidos nessas pesquisas indicam que a maioria dos participantes formou classes de equivalência e que os alimentos e bebidas relacionados, de alguma maneira, com fotos ou expressões faciais denotativas de felicidade foram avaliados como mais agradáveis que aqueles relacionados com fotos ou expressões que representavam tristeza, isso foi evidenciado tanto pela formação de classes de equivalência quanto pela transferência de função. Como afirma Santos (2017, p. 37), o "modelo de equivalência de estímulos possui procedimentos que podem auxiliar no estudo de como as atitudes podem ser formadas ou, até mesmo, modificadas experimentalmente".

Com a finalidade de investigar em quais condições um símbolo abstrato pode adquirir funções simbólicas de personagens e influenciar escolhas alimentares de crianças, Santos (2017) realizou três estudos dos quais participaram crianças com idade entre cinco e seis anos. No Estudo 1, as crianças formaram duas classes de estímulos contendo uma um personagem de que gostavam e a outra um personagem de que não gostavam. Os demais membros da classe eram figuras geométricas e símbolos abstratos. Foram conduzidos três testes de escolha e preferência com dois alimentos idênticos, oferecidos em embalagens transparentes, com os seguintes rótulos: um símbolo equivalente ao personagem favorito e um outro, ao personagem não atrativo; 2) um símbolo equivalente ao personagem não atrativo e um símbolo novo; e 3) um símbolo equivalente ao personagem favorito e um outro, uma marca conhecida. Os resultados mostraram que a maioria das crianças escolheu e relatou gostar mais do alimento com um símbolo da mesma classe do personagem de que gostava. Ainda, o alimento com o símbolo novo no rótulo também foi mais escolhido do que o 
que continha o símbolo da classe do personagem de que não gostavam. O terceiro teste foi inconclusivo. O Estudo 2 foi semelhante ao Estudo 1, mas a marca conhecida no Teste 3 foi substituída por outro símbolo novo. Os resultados do Estudo 2 replicaram o do Teste 1, porém, os Testes 2 e 3 foram inconclusivos. Constatou-se a manutenção das relações de equivalência e transferência de função. No Estudo 3, as crianças formaram três classes de equivalência, em uma das quais havia uma figura neutra. Os testes foram semelhantes aos anteriores, no entanto, os símbolos novos foram substituídos pelo símbolo "neutro". Todas as crianças fizeram sua escolha demonstrando preferência pelo alimento com o símbolo equivalente ao personagem favorito no rótulo. As crianças tendiam a escolher o alimento com um símbolo equivalente ao personagem, mesmo que não atrativo, se a outra opção era um rótulo com um símbolo "neutro". Os resultados sugerem o emprego do paradigma de equivalência como um modelo útil para o estudo das atitudes e preferências.

Evidencia-se que todas as pesquisas supracitadas utilizaram programas de computador ou cartões com figuras ou nomes impressos para a coleta dos dados, mesmo aquelas em que os participantes eram crianças (Santos, 2017; Smeets \& Barnes-Holmes, 2003). Apesar da bem documentada eficácia de ferramentas tradicionais como cartões, cadernos e softwares para o ensino de relações condicionais, quanto mais jovens são os participantes das pesquisas, mais atrativa deve ser a tecnologia empregada para a condução das sessões, já que a repetição de tentativas e o alto número delas - o que geralmente acontece no treino de relações condicionais - podem levar as crianças a se cansarem da tarefa (Straatmann, 2008).

Em razão dos resultados das pesquisas que empregaram jogos para o ensino de habilidades acadêmicas (Siqueira, Barros, Marques, \& Monteiro, 2011; Gris, Alves, Assis, \& Souza, 2017; Xander, Haydu, \& Souza, 2016) e de pesquisas baseados no modelo de equivalência de estímulos e transferência de função, cujo foco é o comportamento alimentar, (Santos, 2017; Cardoso, 2007; Smeets \& Barnes-Holmes, 2003; Straatmann, 2008; Viana, 2006), Panosso e Souza (2014) desenvolveram o jogo Cestinha Mágica, baseado no modelo de equivalência de estímulos, e realizaram uma pesquisa que investigou o efeito do uso do jogo na formação de classes de equivalência, avaliação e escolhas alimentares, realizada por crianças que fazem seleção restritiva alimentar. Participaram duas crianças com idade de sete e oito anos. A pesquisa foi composta por seis etapas: investigação sobre os hábitos alimentares, pré-teste de avaliação e das escolhas alimentares, intervenção/jogo, ensino do procedimento Matching to Sample (MTS), teste das relações emergentes e verificação/pós-teste. Foram conduzidas oito sessões de intervenção com o jogo.

A investigação dos hábitos alimentares era constituída por uma entrevista com os responsáveis pelos participantes e por registro dos alimentos consumidos nas refeições, realizadas por eles, durante três dias. No Pré-teste de Avaliação dos Alimentos, foi apresentada uma folha com 30 figuras, as mesmas usadas no jogo de tabuleiro. Ao lado de cada figura, havia três parênteses e, acima de cada um deles, figuras esquemáticas (smile) que representavam "gostar", "gostar parcialmente" e "não gostar", respectivamente. Os participantes deveriam marcar um $X$ dentro dos parênteses que correspondessem a sua preferência pelo alimento indicado pela figura. No Pré-teste das Escolhas Alimentares eram apresentadas as mesmas figuras do Teste de Avaliação, e os participantes deveriam marcar um X nos parênteses em frente à figura de seis alimentos que escolheriam para comer, caso lhes fossem oferecidos. 
$\mathrm{Na}$ intervenção, usou-se um jogo de tabuleiro para ensinar as relações $A B$ e AC com três conjuntos de estímulos. O Conjunto A - cestas coloridas (cesta de cor laranja - A1, cesta amarela - A2 e a cesta azul - A3), o Conjunto B - alimentos (grupo verduras/legumes/frutas - B1, grupo grãos - B2 e grupo calorias vazias - B3) e o Conjunto C - diferentes quantidades de mãos assinalando positivo (figura de três mãos - C1, duas mãos - C2 e uma mão com os dedo(s) polegar(es) apontado(s) para cima - C3). Após a segunda e a quinta partida do jogo, foram realizados os testes das relações $A B / A C$ e, após oito partidas do jogo, os testes das propriedades emergentes (AA/BB/CC/BA/CA/BC/CB). Os testes foram conduzidos por meio do software de computador Power Point, manuseado pela pesquisadora, e a tarefa dos participantes era tocar na figura que estava na parte de cima da tela e, em seguida, apontar para uma das três figuras apresentadas como estímulos de comparação. Após a realização de duas partidas de jogo, realizou-se a sessão de Verificação 1, após cinco partidas, a Verificação 2 e, após oito partidas, o Pós-Teste. As sessões de Verificação e Pós-teste eram semelhantes às sessões de Pré-teste.

Os resultados mostraram que houve formação de classes de estímulos equivalentes para ambos os participantes, contudo apenas para um deles houve mudanças na avaliação e escolhas alimentares. Esse dado indica que a formação de classes equivalentes por meio do jogo pareceu não afetar as escolhas alimentares dos participantes. Finalmente, ambas as mães relataram que seus filhos experimentaram alimentos sem que elas o exigissem, embora esse dado tenha de ser considerado de maneira cuidadosa, uma vez que partiu do relato das mães e não de observações sistemáticas da pesquisadora. Conquanto o relato das mães indique mudanças no comportamento das crianças, os resultados nos testes de avaliação e escolhas alimentares sugere a necessidade de revisão do procedimento empregado. Por fim, discutem-se outras variáveis do jogo que podem ter promovido as escolhas dos participantes, por exemplo, sentenças escritas compostas por comportamento verbal autoclítico e o emprego de consequências reforçadoras específicas (diferentes quantidades de pontos para a seleção de alimentos de diferentes grupos alimentares). Como a formação de classes de equivalência de estímulos não afetou os resultados das escolhas dos participantes no estudo supracitado, as autoras sugerem que estudos futuros investiguem melhor a interferência das instruções dadas durante o jogo, os efeitos do uso de estímulos reforçadores específicos e de operantes verbais autoclíticos sobre as respostas de consumo alimentar. Aponta-se também a necessidade de replicações do estudo conduzido por Panosso e Souza (2014), a fim de averiguar se os resultados obtidos naquele estudo são obtidos com outros participantes.

A presente pesquisa teve por objetivo, então, investigar os efeitos do jogo de tabuleiro "Cestinha Mágica" sobre as respostas de seleção e consumo de alimentos de diferentes grupos alimentares. Ressalta-se que o trabalho de Panosso e Souza (2014) foi usado como base para esta pesquisa, contudo, algumas alterações dos parâmetros originais daquela pesquisa foram realizadas, entre as quais se destacam: mudanças dos grupos alimentares, mudanças nas regras do jogo, redução da quantidade de cartas com autoclíticos, mudanças nas regras de algumas casas do jogo, observação e registro do consumo alimentar e retirada dos testes de equivalência. Todas as alterações realizadas serão descritas detalhadamente na seção Método. 


\section{Método}

\section{Participantes}

Três crianças com idade entre 5 e 6 anos que, de acordo com relato dos pais e dos profissionais da creche, não comiam alguns tipos de alimentos. As três foram indicadas pelas professoras da creche onde estudavam e por elas eram, diariamente, acompanhadas durante as refeições. Foram excluídas da pesquisa crianças que possuíam intolerância ou eventos passados de situação aversiva com algum alimento, indícios de desenvolvimento precoce de comportamentos de anoréxicos e/ou bulímicos ou de sintomas de depressão infantil bem como crianças que consumiam todos os tipos de alimentos servidos na creche. Esses dados foram obtidos por meio de entrevistas realizadas com os responsáveis pelos participantes. Para efetivar a participação das crianças no estudo, os pais assinaram o Termo de Consentimento Livre e Esclarecido (TCLE). Este trabalho foi aprovado pelo Comitê de Ética com Humanos, parecer número 109/2012.

\section{Local, Materiais e Equipamentos}

A coleta de dados foi realizada em uma creche da cidade de Londrina, PR, em uma sala disponibilizada pela diretoria. Foram usadas para a coleta uma mesa, duas cadeiras, caneta, lápis e prancheta, uma entrevista semiestruturada pré e pós-intervenção, folha de registro do consumo alimentar, folha de registro das jogadas, jogo de tabuleiro "Cestinha Mágica". A entrevista semiestruturada era composta de 39 questões que incluíam informações sobre dados pessoais (idade, sexo, problemas de saúde etc.), dados sobre a rotina alimentar (horários das refeições, alimentos servidos etc.) e sobre o comportamento alimentar (quem serve a criança, o que acontece se ela não come, o que acontece se ela come etc.).

Os equipamentos utilizados foram uma câmera filmadora e um notebook.

\section{Jogo de tabuleiro Cestinha Mágica (Panosso \& Souza, 2014)}

O jogo é composto por um tabuleiro, três peões, 150 pontos em forma de estrela (50 laranjas, 50 amarelas e 50 azuis), um dado com números de um a três, 60 cartas, duas foIhas-matriz e um Manual de Instruções.

Folha-matriz. Em uma folha A4, era apresentada uma tabela com as figuras de uma cesta laranja, uma cesta amarela e uma cesta azul ao lado das quais os cartões sorteados durante o jogo deveriam ser colocados, possibilitando o emparelhamento do alimento com a cesta.

Tabuleiro. 57 casas; a casa da partida, 18 casas com figuras de cestas de supermercado, seis com figura de uma cesta de cor laranja, seis com a figura de cesta de cor amarela e seis com a figura de cesta de cor azul. Havia, ainda, 24 casas com a imagem que agrupava cestas de supermercado laranja, amarelas e azuis e figuras de mão(s) indicando gesto de positivo em uma mesma carta, sendo: seis casas com a figura de uma cesta de cor laranja e três figuras de mãos com o dedo polegar apontado para cima (indicando gesto de positivo); seis com a figura de uma cesta de cor amarela e duas mãos com o dedo polegar apontado para cima e seis com a figura de uma cesta de cor azul e uma figura de uma mão com dedo polegar apontado para cima. O tabuleiro era composto também por três casas com a figura de um bruxo, três casas sobre as quais consta a frase "casa da cesta vazia" impressa sobre elas, três 
com a frase "casa da troca", três casas com uma figura de uma cesta branca, uma casa da cesta mágica e uma casa da chegada.

Cartas. O jogo foi composto de 60 cartas divididas da seguinte maneira: 48 cartas em cuja Face A havia a figura de uma cesta e sobre a Face B, a figura de um alimento; seis cartas-bônus, em cuja Face A havia a figura de uma cesta e na Face B, uma frase; e seis cartas do bruxo em cuja Face A havia a figura do bruxo e na Face B, uma frase. As figuras dos alimentos selecionados para esta pesquisa referem-se apenas àqueles que eram oferecidos aos participantes na instituição onde foi realizada a pesquisa. A Figura 1 apresenta o jogo de tabuleiro, figura do bruxo apresentada no jogo em formato tridimensional e cartas que o compõem.

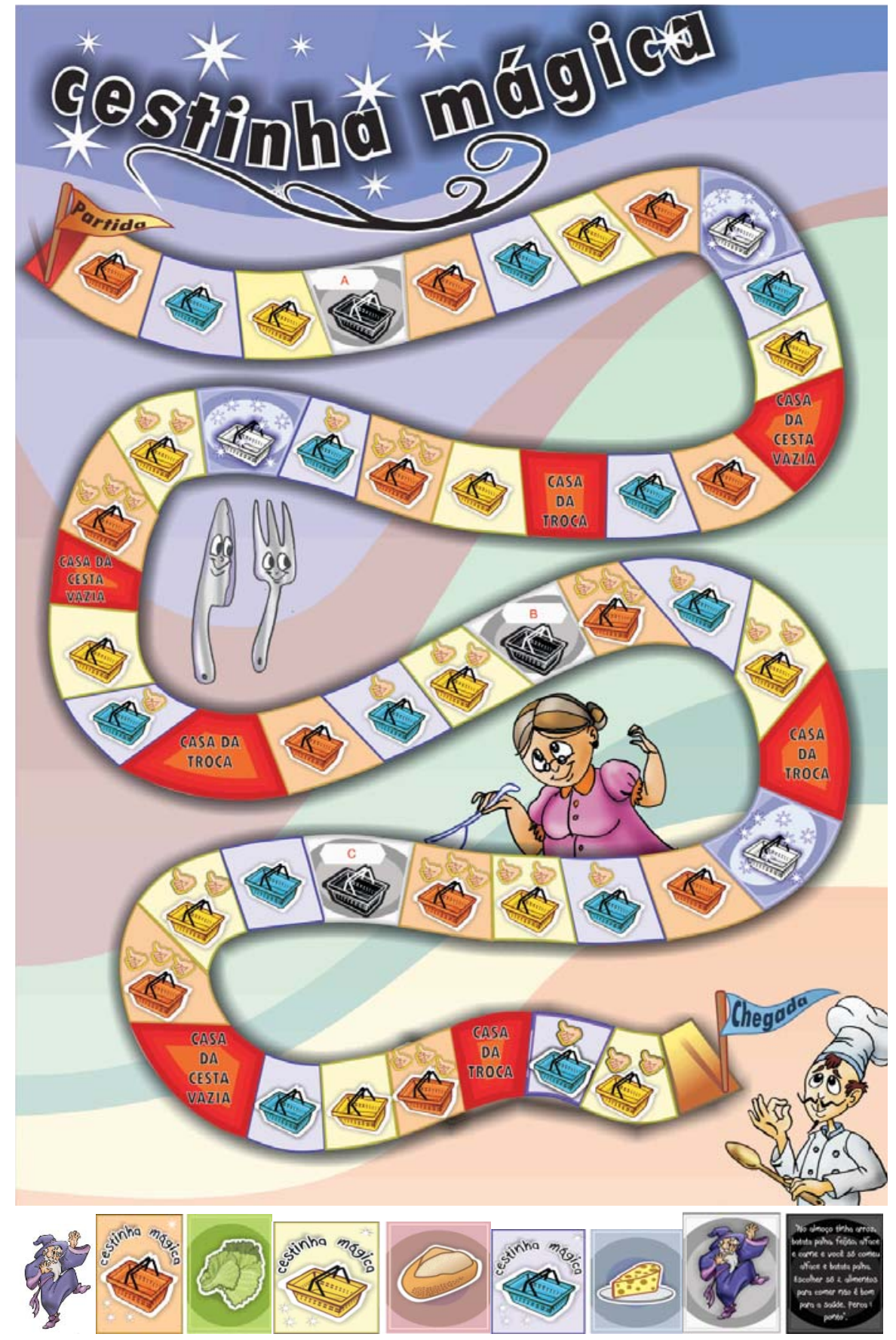

Figura 1. Jogo de tabuleiro, figura do bruxo apresentada no jogo em formato tridimensional e exemplos de cartas que o compõem. 


\section{Estímulos Experimentais}

As casas e as cartas do jogo possibilitam o ensino da relação entre três conjuntos de estímulos: as cestas coloridas (Conjunto A), os alimentos (Conjunto B) e as figuras de mãos com o dedo polegar apontado para cima, indicando positivo em quantidades diferentes (Conjunto C). Os Conjuntos A e C possuíam três elementos cada, e o Conjunto B, 24 elementos.

O jogo trabalha com os três grupos alimentares, indicados para o consumo diário: verduras/legumes/frutas, cereais/grãos e proteínas/ laticínios. Os grupos verduras, legumes e frutas assim como o de proteínas e laticínios foram considerados, na presente pesquisa, como um único grupo alimentar para facilitar a confecção do jogo. De acordo com as recomendações do Dietary Guidelines e MyPlate (http://www.usda.gov/wps/portal/usda/usdahome), uma pessoa deve ingerir metade de um prato de refeição em alimentos dos grupos verduras/legumes/frutas, mais de um quarto de alimentos do grupo dos cereais/grãos e um quarto de proteínas. As pontuações atribuídas no jogo seguiram essas orientações.

Elaboração do jogo. Para compor o jogo foram selecionados alimentos que fazem parte da rotina diária da família brasileira, conforme informações apresentadas pelo Ministério da Saúde do Governo Brasileiro - Portal da Saúde (2006). Para esta pesquisa, foram utilizadas apenas figuras de alimentos dos grupos das verduras/legumes/frutas, cereais/grãos e proteínas/laticínios disponíveis na refeição do almoço do local onde foi realizada a pesquisa. Serviu de informação o cardápio oferecido às crianças durante o período da realização da pesquisa. O jogo não trabalha com o critério de quantidade e/ou porções diárias indicadas para o consumo de cada grupo alimentar, pois nesta pesquisa não se visa o ensino de condutas alimentares relacionadas a quantidades, horário das refeições e balanceamento nutricional personalizado. A Figura 2 apresenta o manual do jogo que contém seu objetivo, a forma de jogar e os critérios para ser o vencedor. 


\section{Manual do Jogo}

OBJETIVO: Ter mais fichas e variedade de figuras de alimentos quando um dos participantes chegar ao final do jogo.

PREPARAÇÃO PARA O JOGO: abra o tabuleiro e distribua em frente ao mesmo as cartas referentes aos alimentos da seguinte forma: junte todas as cartas que possuem as mesmas cores de cestas de supermercado. Distribua em três montes, um dos quais o das cartas de cestas de cor laranja, um das cestas amarelas e outro de cestas azuis, e embaralhe as cartas em cada monte. Junte ainda as cartas que possuem o desenho do bruxo, forme outro monte e coloque-o ao lado dos outros três montes de cartas. As cartas deverão ser embaralhadas e apresentadas com a face que possui o desenho da cesta e do bruxo virados para cima para que todos possam ver. Distribua uma folha-matriz para os participantes. Em seguida, cada jogador deverá escolher a cor do peão com o qual irá jogar. COMO JOGAR: para iniciar o jogo, cada jogador deverá lançar o dado. Quem tirar o número maior dará início à partida lançando o dado novamente e percorrendo o número de casas correspondente ao número do dado.

Se o peão parar em uma casa com a figura de uma cesta laranja, o participante deverá pegar a primeira carta do monte de cesta laranja. Se ele tirar uma carta com a figura de uma fruta, verdura ou legume, ganhará três estrelas (três pontos) e se sortear uma carta com a frase carta-bônus: ganhe 1 ponto e pegue a próxima carta, deverá executar esta tarefa e ganhar o número de pontos correspondentes às cartas sorteadas. Se o peão parar na casa da cesta amarela, o participante deverá pegar a primeira carta do monte de cesta amarela. Se ele tirar uma carta com a figura de um alimento do grupo dos cereais/grãos, ganhará duas estrelas (dois pontos) e se sortear uma carta com a frase carta-bônus: ganhe 1 ponto e pegue a próxima carta, deverá executar essa tarefa e ganhar o número de pontos correspondentes às cartas sorteadas. Se o peão parar na casa da cesta azul, o participante deverá escolher uma carta do monte da cesta azul. Se sortear uma carta com um alimento do grupo das proteínas/laticínios, ganhará uma estrela (um ponto), e se sortear uma carta com a frase carta-bônus: ganhe 1 ponto e pegue a próxima carta, deverá executar essa tarefa e ganhar o número de pontos correspondentes às cartas sorteadas. As cartas de alimentos deverão ser posicionadas nas folhas-matriz em frente à cor das cestas correspondentes aos alimentos. Se o peão parar na casa de cesta de cor laranja com três mãos com o dedo polegar virado para cima, automaticamente ganhará três estrelas (pontos) do jogo. Se o peão parar na casa de cesta amarela com duas mãos com o dedo polegar virado para cima, ganhará duas estrelas (pontos) do jogo, e se cair na casa de cesta azul com uma mão com o dedo polegar está virado para cima, ganhará uma estrela (ponto) do jogo. A cor dos pontos será referente à cor da cesta. Se o peão parar na "casa da cesta vazia", deverá avançar uma casa. Se parar na "casa da troca", deverá trocar um de seus alimentos, e os pontos correspondentes, por qualquer alimento do outro jogador. Se parar na "casa da cesta mágica", o participante poderá jogar mais duas vezes, caso tenha folha-matriz pelo menos um alimento de cada cestinha. Caso contrário, deve esperar a próxima jogada. Se o peão parar na "casa do bruxo", o participante deverá sortear uma carta do monte de cartas do bruxo e seguir as instruções fornecidas na carta sorteada.

VENCEDOR: vence o jogo quem tiver o maior número de pontos no final do mesmo, independentemente de quem chegar ao final primeiro, e se tiver na folha-matriz pelo menos um alimento em cada cestinha. Caso contrário, o jogador perderá cinco pontos e os dois jogadores deverão contar os pontos novamente.

Figura 2. Manual de Instruções do Jogo. 


\section{Procedimento}

Após assinatura do TCLE, realizou-se a primeira entrevista para conhecer o histórico alimentar do participante e hábitos familiares relacionados à refeição. Em seguida, iniciaram-se as observações da refeição do almoço realizada no ambiente escolar. A observação e o registro dos alimentos consumidos pelos participantes foram realizados diariamente, durante 45 dias, ao longo de todas as fases do estudo (linha de base, intervenção e pós-intervenção). Os dias de observação e registro eram alternados entre a pesquisadora e uma auxiliar de pesquisa devidamente treinada. A pesquisa empregou o Delineamento Experimental de Sujeito Único - Linha de Base Múltipla entre sujeitos, de maneira que a coleta de dados teve início com o P1. Após três partidas com o jogo com o P1, deu-se início às partidas com o P2 e, após seis partidas com o P2, iniciaram-se as partidas com o P3. Uma descrição mais detalhada de cada uma das três fases do estudo encontra-se abaixo.

Fase de linha de base. Nesta fase, registraram-se todos os alimentos escolhidos e consumidos pelos participantes (etapa de observação do consumo alimentar - O.C.). As refeições eram servidas no prato das crianças, em pequenas porções, dentro da cozinha, sem que a elas tivessem acesso ou pudessem escolher os alimentos que gostariam de consumir. Nenhuma instrução foi dada à cozinheira ou às crianças. Isso foi feito para que se observassem a rotina da creche no referente ao horário do almoço e o comportamento das crianças. Após a etapa O.C., figuras dos alimentos que seriam servidos no almoço eram apresentadas aos participantes, dando-se the a seguinte instrução: "Estes são os alimentos que temos para o almoço de hoje (apresentavam-se as figuras dos alimentos). Quais você escolhe para comer?". A informação sobre as escolhas era repassada à cozinheira, que colocava no prato de cada participante os alimentos escolhidos, independentemente do grupo alimentar, i.e., o participante poderia escolher, para comer, alimentos de apenas um grupo alimentar. Essa atividade era realizada dez minutos antes do início da refeição e, em seguida, observava-se se os alimentos escolhidos eram ingeridos (etapa de escolha dos alimentos por meio de figuras e observação do consumo alimentar - E.O.C.). Todas as refeições do almoço eram feitas em grupo, conforme a rotina da escola.

Fase de intervenção/jogo. Para cada participante realizou-se uma partida de jogo por sessão. Cada partida durou, em média, 30 minutos e era realizada apenas entre a pesquisadora e um participante. Durante o jogo, a pesquisadora instruía apenas quando era solicitada a fazê-lo pelo participante. Todas as instruções referiam-se às regras de funcionamento do jogo. Uma partida se iniciava quando o dado era lançado pela primeira vez e finalizava quando o dado era jogado pela última vez antes da contagem dos pontos obtidos por cada jogador. Para iniciar o jogo, as cartas eram embaralhadas e distribuídas em quatro montes: cartas com as figuras das cestas de cor laranja, cartas das cestas amarelas, cartas das cestas azuis e cartas com a figura do bruxo. Em seguida, a pesquisadora explicitava ao participante as regras do jogo, contidas no Manual de Instruções, e o convidava a jogar. Cada jogador lançava o dado para decidir quem iniciaria o jogo. Quem tirasse o número maior iniciaria a partida lançando o dado novamente e percorrendo o número de casas correspondente ao número apresentado pelo dado. Os participantes foram submetidos às sessões de jogo de duas a três vezes por semana. 
Fase pós-intervenção. Ao finalizar a fase de intervenção, realizou-se a fase das escolhas alimentares por meio das figuras e a observação do consumo alimentar (E.O.C), e a fase de observação do consumo alimentar (O.C.) sem que houvesse a escolha dos alimentos antes das refeições. Nessa fase, contudo, a cozinheira foi orientada a servir o prato com todos os alimentos disponíveis. Essa mudança em relação à fase de linha de base tinha por finalidade permitir avaliar o consumo dos alimentos servidos, já que, em razão das regras da creche, os alimentos eram servidos no prato, sem que a criança pudesse selecionar o que gostaria de comer.

Após a conclusão de todas as fases, os responsáveis pelos participantes foram entrevistados individualmente, para informar se houve alguma mudança no comportamento alimentar do participante em casa. Repassou-se também uma devolução dos resultados da pesquisa e orientou-se sobre como lidar com o comportamento alimentar de cada participante.

\section{Resultados}

De acordo com as entrevistas realizadas com os responsáveis, o P1 consumia somente alguns tipos de legumes/verduras (repolho e alface). Quanto às proteínas, às vezes comia carne. Não tomava leite. Quando o P1 não queria consumir os alimentos oferecidos, o responsável permitia que ele consumisse outros alimentos de sua preferência. O P2 não consumia verduras e frutas. Em razão disso, muitas vezes seus responsáveis o castigavam (sem jogar videogame ou sem fazer visitar à avó). Contudo, se o P2 solicitasse um alimento que o responsável não julgasse adequado (e.g., um doce fora de hora), ele permitia o consumo. 0 P3 recusava-se a consumir verduras, legumes e a carne nas refeições servidas em casa. Ele tinha sido amamentado até poucos meses antes do início da pesquisa. A responsável pelo P3 relatou que oferecia alimentos que a criança gostava de consumir e que retirava do prato alimentos que ele não queria consumir. O P1 e o P3 comiam bolacha recheada no café da manhã, antes de ir para a creche. De acordo com os dados fornecidos nas entrevistas, as crianças se recusavam a comer determinados tipos de alimentos (e.g., legumes, verduras) em qualquer situação e na presença de qualquer pessoa. Ressalta-se que nenhum dos participantes apresentava problemas de saúde que se poderiam atribuir ao seu padrão alimentar, de acordo com relato dos pais e professores.

\section{Fase de linha de base}

De modo geral, nessa fase eram servidos para as crianças apenas alimentos que elas tinham por hábito consumir, e não todos aqueles que estavam disponíveis. De acordo com a cozinheira, isso era feito para evitar desperdícios. Ressalta-se que a creche oferecia pelo menos duas alternativas de cada grupo alimentar por dia. Na etapa O.C. (observação do consumo alimentar) foram oferecidos dois tipos de alimentos do grupo dos cereais/grãos (arroz e macarrão), quatro tipos de proteína (feijão, ovo, frango e carne), quatro tipos de frutas (tomate, maçã, banana e melancia) e dois tipos de verduras/legumes (repolho e pepino). Para o P2, devido à falta na instituição, foi oferecido apenas um tipo de alimento do grupo verduras e legumes (pepino), dois do grupo dos cereais/grãos e de proteínas (feijão e frango) e três tipos de frutas. Com o P1 foram realizadas três sessões de O.C., com o P2 duas sessões, devido a suas faltas à instituição, e com o P3 três. 
Foram realizadas seis sessões da etapa E.O.C. (escolha dos alimentos por meio de figuras e observação do consumo alimentar) com o P1, oito com o P2 e nove com o P3. Nessa etapa, foram oferecidos aos participantes os seguintes alimentos: alface, acelga, repolho, pepino, brócolis, couve-flor (grupo das verduras/legumes) e tomate, maçã, abacaxi e melão (do grupo das frutas) arroz, macarrão, nhoque e batata (grupo dos cereais/grãos) frango, carne moída e em pedaços, ovos e feijão (grupo das proteínas). Todos esses alimentos não estavam disponíveis diariamente em todas as sessões. Houve variação dos alimentos dentro de um mesmo grupo alimentar, de acordo com cardápio do dia ofertado pela escola. A Tabela 1 apresenta os alimentos disponíveis nesta fase e aqueles que foram consumidos pelas crianças.

\section{Tabela 1}

Alimentos de cada grupo alimentar oferecidos e consumidos pelos participantes do estudo (P1, P2 e P3) na fase de linha de base (LB), intervenção (I) e pós-intervenção (PI).

\begin{tabular}{|c|c|c|c|c|c|c|c|c|c|c|c|c|c|}
\hline \multirow{3}{*}{ Grupo } & \multirow{3}{*}{ Alimento } & \multicolumn{4}{|c|}{ P1 } & \multicolumn{4}{|c|}{ P2 } & \multicolumn{4}{|c|}{ P3 } \\
\hline & & \multicolumn{2}{|c|}{ LB } & \multirow{2}{*}{1} & \multirow{2}{*}{ PI } & \multicolumn{2}{|c|}{ LB } & \multirow{2}{*}{$\mathbf{I}$} & \multirow{2}{*}{ PI } & \multicolumn{2}{|c|}{ LB } & \multirow{2}{*}{ I } & \multirow{2}{*}{ PI } \\
\hline & & OC & EOC & & & $O C$ & EOC & & & OC & EOC & & \\
\hline \multirow{6}{*}{$\begin{array}{l}\text { Verduras } \\
\text { e legumes }\end{array}$} & Repolho & $x$ & $x$ & $\otimes$ & $\otimes$ & - & $\otimes$ & $\otimes$ & & $\otimes$ & $\otimes$ & $\otimes$ & $\otimes$ \\
\hline & Pepino & $x$ & $x$ & $x$ & $x$ & $\otimes$ & $x$ & $\otimes$ & & $\otimes$ & $\otimes$ & $\otimes$ & $\otimes$ \\
\hline & Alface & - & $\otimes$ & $\otimes$ & $\otimes$ & - & $\otimes$ & $\otimes$ & & - & $\otimes$ & $\otimes$ & $x$ \\
\hline & Acelga & - & $x$ & - & - & - & $\otimes$ & - & & - & & - & - \\
\hline & Brócolis & - & - & - & - & - & $x$ & $\otimes$ & & - & $\otimes$ & $\otimes$ & - \\
\hline & Couve-flor & - & - & - & - & - & $x$ & - & & - & $\otimes$ & - & - \\
\hline \multirow{6}{*}{ Frutas } & Tomate & $x$ & $x$ & $\otimes$ & $\otimes$ & $\otimes$ & $\otimes$ & $\otimes \otimes$ & & $\otimes$ & $\otimes$ & $\otimes$ & - \\
\hline & Maça & $\otimes$ & $\otimes$ & $\otimes$ & $\otimes$ & - & $\otimes$ & $\otimes$ & & $\otimes$ & $\otimes$ & $\otimes$ & $\otimes$ \\
\hline & Melão & - & $\otimes$ & $\otimes$ & $\otimes$ & - & $\otimes$ & $\otimes$ & & - & $\otimes$ & $\otimes$ & $\otimes$ \\
\hline & Abacaxi & - & $\otimes$ & $\otimes$ & $\otimes$ & - & $x$ & $\otimes$ & & - & $\otimes$ & $\otimes$ & $\otimes$ \\
\hline & Melancia & $\otimes$ & $\otimes$ & - & - & $\otimes$ & $\otimes$ & $x$ & & $x$ & $\otimes$ & $\otimes$ & - \\
\hline & Banana & $\otimes$ & $\otimes$ & $\otimes$ & $\otimes$ & $\otimes$ & $\otimes$ & $\otimes$ & & $x$ & $\otimes$ & $\otimes$ & - \\
\hline \multirow{5}{*}{ Proteínas } & Feijão & $x$ & $x$ & $x$ & $x$ & $\otimes$ & $\otimes$ & $\otimes$ & & $\otimes$ & $\otimes$ & $\otimes$ & $\otimes$ \\
\hline & Ovo & $\otimes$ & $\otimes$ & $\otimes$ & $\otimes$ & - & $x$ & $x$ & & $x$ & $\otimes$ & $\otimes$ & $x$ \\
\hline & Frango & $\otimes$ & $x$ & $\otimes$ & $\otimes$ & $\otimes$ & $\otimes$ & $\otimes$ & & $x$ & $x$ & $x$ & $x$ \\
\hline & Carne moída & $x$ & $x$ & $\otimes$ & $x$ & $\otimes$ & $\otimes$ & $\otimes$ & & $x$ & $x$ & $\otimes$ & - \\
\hline & Carne em pedaços & - & $x$ & $\otimes$ & $\otimes$ & - & $\otimes$ & $\otimes$ & & - & $x$ & $x$ & $x$ \\
\hline \multirow{4}{*}{$\begin{array}{l}\text { cereais/ } \\
\text { grãos }\end{array}$} & Arroz & $\otimes$ & $\otimes$ & $\otimes$ & $\otimes$ & $\otimes$ & $\otimes$ & $\otimes$ & & $\otimes$ & $\otimes$ & $\otimes$ & $\otimes$ \\
\hline & Macarrão & $x$ & $x$ & $\otimes$ & $\otimes$ & - & $\otimes$ & $\otimes$ & & $x$ & $x$ & $\otimes$ & - \\
\hline & Batata & - & $x$ & $\otimes$ & - & - & $x$ & $\otimes$ & & - & $\otimes$ & $\otimes$ & - \\
\hline & Nhoque & - & $x$ & - & - & - & $\otimes$ & - & & - & $x$ & - & - \\
\hline \multicolumn{14}{|c|}{$\begin{array}{l}\text { Nota. A abreviação LB refere-se à fase observação do consumo alimentar durante a refeição do almoço e E.O.C. } \\
\text { fase de escolhas dos alimentos por meio das figuras e a observação do consumo alimentar durante a refeição } \\
\text { do almoço. I refere-se à fase de intervenção e PI à fase de pós-intervenção. O X refere-se aos alimentos ofereci- } \\
\text { dos, mas não consumidos pelo participante, } \otimes \text { refere-se aos alimentos oferecidos e consumidos e (-) refere-se } \\
\text { a alimentos que não foram apresentados naquela fase. }\end{array}$} \\
\hline
\end{tabular}


Observa-se que todas as crianças consumiam pelo menos um alimento de cada grupo. 0 P3 foi o participante que consumiu a maior variedade de alimentos servidos. Ele consumiu todas as verduras, legumes e frutas oferecidos, recusando-se a comer apenas alguns alimentos do grupo das proteínas e cereais/grãos.

Fase de intervenção/jogo. Nessa fase, o P1 obteve oito vitórias e um empate em 12 partidas; o P2 e o P3 obtiveram duas vitórias e dois empates, respectivamente em 11 e 13 partidas. Durante as partidas, o dado foi lançado 46 vezes. O número de partidas variou devido às faltas dos participantes à creche. A Tabela 2 apresenta os emparelhamentos entre cartas e alimentos mais frequentes para cada participante.

\section{Tabela 2}

Emparelhamentos entre cartas e alimentos mais frequentes para cada participante.

\begin{tabular}{|c|c|c|c|c|}
\hline Part. & Relações & $\begin{array}{l}\text { № de vezes } \\
\text { em que houve } \\
\text { emparelhamento da } \\
\text { carta com alimento }\end{array}$ & Relações & $\begin{array}{l}\text { № de vezes } \\
\text { em que houve } \\
\text { emparelhamento da } \\
\text { carta com alimento }\end{array}$ \\
\hline \multirow{3}{*}{ P1 } & Cesta laranja-abacaxi & 9 & Cesta azul-queijo & 3 \\
\hline & Cesta laranja-brócolis & 7 & Cesta azul-feijão & 3 \\
\hline & & & Cesta azul-frango & 3 \\
\hline \multirow{3}{*}{ P2 } & Cesta laranja-alface & 10 & Cesta laranja-maçã & 2 \\
\hline & Cesta amarela-batata & 8 & Cesta laranja-tomate & 2 \\
\hline & Cesta azul-queijo & 8 & Cesta azul-requeijão & 2 \\
\hline \multirow{4}{*}{ P3 } & & & Cesta azul-carne & 2 \\
\hline & Cecta larania-tomato & $\begin{array}{c}10 \\
9\end{array}$ & Cesta azul-queijo & 3 \\
\hline & Cesta & 9 & Cesta azul-requeijão & 3 \\
\hline & cesta d $\angle$ i-logurte & $y$ & Cesta laranja-banana & 3 \\
\hline
\end{tabular}

Em relação às demais casas do jogo, o peão do P1, do P2 e do P3 parou 16, 22 e 19 vezes, respectivamente, na casa da cesta mágica. Na casa da troca, o peão do P1 parou 19 vezes, o do P2 e o do P3, 23 vezes. Na casa do bruxo, o peão do P1 parou 17 vezes, o do P2 parou 19 vezes, e o do $\mathrm{P} 3$ parou 23 vezes.

Quando se analisam apenas os alimentos que foram servidos em todas as etapas do estudo para cada criança, verifica-se que o P1 e o P2, com exceção do P2 no concernente ao grupo das proteínas, consumiram maior variedade de alimentos de cada grupo. O P3 experimentou apenas dois novos alimentos (carne moída e macarrão). É importante lembrar que todos os participantes consumiram alimentos não usuais apenas após o início da intervenção, o que sugere que consumiram alimentos não usuais em razão da intervenção e não por outras variáveis. Ainda, durante a Fase de intervenção foram oferecidos e consumidos outros alimentos não considerados nos dados da Tabela 1. Por exemplo, na fase de intervenção, o P1 consumiu alimentos dos grupos das frutas e das proteínas (manga e bolo de carne), o P2 consumiu alimentos dos grupos dos legumes, das frutas e dos cereais/grãos (beterraba, manga, uva e lasanha) e o P3 alimentos do grupo dos legumes, frutas e cereais/grãos (beterraba, manga e lasanha). 
Fase pós-intervenção. A fase das escolhas alimentares feitas pelas crianças por meio das figuras e a observação do consumo alimentar (E.O.C) foi feita durante sete dias com o P1 e com o P3 durante três dias. Em razão do não comparecimento às aulas, esta fase não foi realizada com o P2. Em seguida, durante mais três dias realizou-se a observação do consumo alimentar do P1 e do P3, sem que houvesse a escolha dos alimentos antes das refeições. Não obstante o consumo de alimentos do grupo dos cereais/grãos pelo P1 ter ficado acima do observado na fase de linha de base, a quantidade dos alimentos desse grupo oferecida teve redução, quando se comparam as sessões desta fase com as anteriores (fase de intervenção). O P3 também deixou de consumir alguns alimentos dos grupos das verduras/legumes e das proteínas/laticínios que havia consumido nas fases anteriores.

Resumindo, tanto os dados da entrevista inicial com os profissionais da creche e com os pais quanto as observações iniciais sugerem que, embora as crianças não apresentassem restrições alimentares severas, elas davam preferência a alguns tipos de alimentos refugando outros; eram mais seletivas para as escolhas relacionadas ao grupo dos legumes e verduras (P1 e P2), proteínas (P1 e P3) e cereais/grãos (P1). Após o início da intervenção, o P1 consumiu maior variedade de verduras/legumes, proteínas e cereais/grãos, quando se faz a comparação entre a fase de linha de base e a da intervenção. Apenas a qualidade e a quantidade de verduras/legumes consumidos se mantiveram na fase de pós-intervenção, conquanto o consumo dos alimentos dos demais grupos tenha ficado acima do observado na linha de base. O P2 consumiu principalmente diferentes verduras/legumes e cereais/grãos em relação aos consumidos na linha de base, e o P3 consumiu pelo menos um alimento a mais do grupo das proteínas e cereais/grãos. Não foi possível avaliar se o P3 manteve o consumo desses alimentos, uma vez que estes não foram oferecidos na pós-intervenção.

Quanto à entrevista final, todos os responsáveis pelas crianças relataram ter observado mudança no comportamento alimentar de seus filhos. O responsável pelo P1 relatou haver observado o consumo de maior quantidade de verduras e carnes em relação à fase anterior à pesquisa. O responsável pelo $\mathrm{P} 2$ relatou que, antes da intervenção, o P2, em casa, consumia apenas arroz, feijão e carne e, após a intervenção, passou a consumir qualquer tipo de verdura, legume e fruta que the oferecessem. Cabe ressaltar que, de acordo com as observações realizadas na creche, o P2 consumiu quase todas as frutas e verduras que lhe foram oferecidas. Finalmente, a responsável pelo P3 relatou que ele diminuiu o consumo de alimentos como bolacha recheada, balas, chocolate, salgadinhos e aumentou o consumo de iogurtes e sucos de frutas. Os responsáveis pelas crianças relataram ainda que, no período da pesquisa, a rotina alimentar relacionada a horários, local e pessoas envolvidas nas refeições familiares não foi alterada. No período da pesquisa, nenhum dos participantes teve problemas relacionados à saúde física, nem tomaram medicamentos ou receberam orientações de profissionais da saúde para modificarem a alimentação. Além disso, não houve modificações na rotina de alimentação da escola.

\section{Discussão}

A maior variedade de alimentos consumidos após o início da intervenção sugere que o jogo contribuiu para as mudanças observadas e indica a necessidade de uma análise mais cuidadosa das características do jogo que podem ter contribuído para essa mudança de 
comportamento. De acordo com as regras do jogo, para terem maiores chances de ganhar, era necessário que os participantes tivessem em sua folha-matriz alimentos de todos os grupos. A instrução "Vence o jogo quem tiver o maior número de pontos ao final do mesmo, independente de quem chegar ao final primeiro..." especifica a consequência reforçadora (ganhar o jogo). Isso pode ter contribuído para que alguns participantes selecionassem mais alimentos que valiam mais pontos, como os do grupo das verduras/legumes. No entanto, apenas selecionar estes alimentos não garantia a vitória no jogo, porque a segunda parte da instrução descrevia que era necessário ter alimentos de todos os grupos alimentares ao final do jogo, caso contrário, o jogador perderia 5 pontos. A instrução estabeleceu um prazo (final do jogo) para a emissão das respostas de selecionar alimentos variados para evitar a perda de pontos. Assim, a segunda parte da instrução pode ter funcionado como operação que estabeleceu a não variedade de alimentos como condição aversiva.

As contingências de controle aversivo podem funcionar como operações estabelecedoras na medida em que aumentam o valor (reforçador) da suspensão da estimulação aversiva e evocam comportamentos de fuga ou esquiva (Michael, 1993). Após a instrução de selecionar alimentos de todos os grupos, o comportamento do jogador era reforçado negativamente por esquivar-se da consequência aversiva (perder pontos). Os participantes esquivavam-se à perda de pontos selecionando alimentos de todos os grupos alimentares, especialmente quando o peão parava na casa da troca. Abreu e Hübner (2011) também observaram que a instrução com a descrição de consequência aversiva controlou o comportamento-alvo dos participantes desse estudo (respostas de checagem).

Provavelmente, seguir uma instrução que descreve uma condição aversiva pelo seu não-seguimento é resultado de uma história de reforço para seguir regras. Segundo Albuquerque e Paracampo (2010), uma regra é seguida porque seguir regras semelhantes foi reforçado e/ou seu não-seguimento foi punido. A instrução da casa da cesta mágica, que descrevia a consequência reforçadora da seleção de variedade alimentar, também pode ter sido seguida devido a uma história de reforço para o seguimento de instruções.

Outro aspecto refere-se ao fato de as regras fornecidas terem atuado como operações estabelecedoras, alterando a efetividade reforçadora dos pontos. As operações estabelecedoras são variáveis ambientais, operações ou condições de estímulos, que alteram momentaneamente a efetividade reforçadora de outros eventos e a frequência de ocorrência de todo comportamento que foi reforçado por esses eventos (Michael, 1993). A regra inicial estabeleceu que um dos critérios para ganhar o jogo era ter maior quantidade de pontos ao final. Assim, no contexto do jogo, os pontos tiveram sua efetividade reforçadora aumentada e, consequentemente, a ocorrência de classes de respostas que permitiam a obtenção de pontos pode ter tido aumento de frequência. As consequências empregadas no jogo foram consequências reforçadoras específicas relacionadas à quantidade de pontos. Apesar de não ter sido testado o conhecimento dos participantes no respeitante ao conceito "maior que" em relação aos números de pontos (três, dois e um), é possível que, em razão de uma história pré-experimental, os participantes tenham estabelecido, de acordo com Steele e Hayes (1991), uma relação de comparação "maior do que" ou "mais que". No jogo, ganhar três pontos era melhor que ganhar dois pontos e ganhar dois pontos era melhor que ganhar um ponto. Os alimentos emparelhados com os pontos podem, via formação de classes de 
equivalência, ter adquirido a mesma função reforçadora dos pontos. Ainda, de maneira similar ao quadro relacional de comparação estabelecido entre os pontos, é possível que os alimentos também tenham estabelecido esta relação entre si e, consequentemente, os alimentos do grupo das verduras/legumes/frutas podem ter adquirido uma função reforçadora de maior magnitude que a dos alimentos dos demais grupos. Assim, os pontos podem ter transformado a função dos alimentos. Essa poderia ser uma das razões por que o P1 e o P2 consumiram uma maior variedade de alimentos do grupo das verduras/legumes e o P3, variedade maior de frutas após o início da intervenção. No entanto, nenhum teste foi realizado para verificar se ocorreu de fato essa transferência de função, o que mostra a limitação desse estudo. Novas investigações deveriam avaliar essa questão.

As instruções do jogo podem ter ensinado os participantes a aumentar a seleção de variedade de alimentos. Para as escolhas, em ambiente natural, nenhuma instrução, ou consequência, era fornecida. Contudo, é possível que, quando a pesquisadora ou auxiliar de pesquisa apresentava as figuras dos alimentos aos participantes para que escolhessem o que iriam consumir, eles possam, por generalização de estímulos, ter apresentado respostas de selecionar variedade alimentar, pois as figuras dos alimentos eram as mesmas do jogo. Ainda, a presença da pesquisadora ou da auxiliar de pesquisa, no momento da seleção dos alimentos, por meio das figuras, e no momento da refeição, pode ter interferido na escolha e consumo dos alimentos. A presença da pesquisadora ou auxiliar, nessas condições, pode ter funcionado como um estímulo discriminativo para o seguimento de regras (Hübner, Austin, \& Miguel, 2008). Novas investigações a respeito dessa questão poderiam ser feitas de maneira que o encarregado de apresentar as figuras dos alimentos aos participantes, para que procedessem a escolha do que iriam consumir, fosse um auxiliar de pesquisa que não estivesse presente durante o jogo e nem no momento das refeições.

Ainda, observou-se que nem todos os alimentos eram apresentados aos participantes no decorrer das refeições, ou seja, se o participante tinha por hábito não consumir determinado alimento, este deixava de ser oferecido à criança. Após o início da intervenção, observou-se que os participantes passaram a consumir alimentos que não tinham por hábito consumir. Isso pode ter acontecido, pois, ao contrário do que acontecia na linha de base, na fase de intervenção e pós-intervenção, os participantes podiam escolher o que iriam consumir, i.e., escolhiam o que iriam comer por meio das figuras ou todos os alimentos estavam no prato e se deixava a criança livre para consumir o que quisesse como aconteceu na pós-intervenção O.C. O P1, por exemplo, só passou a consumir proteínas e repolho e tomate após o início da intervenção. A presença do alimento no prato ou a possibilidade de fazer a escolha podem ter contribuído para esse resultado, e isso indica que estudos futuros deveriam melhorar o controle experimental no referente a essa questão. O alimento e sua representação (figura) deveriam estar disponíveis para a criança desde o início da intervenção.

Destaca-se que muitos alimentos servidos na fase de linha de base e intervenção não foram servidos na fase de pós-intervenção, o que dificulta afirmar se os pequenos efeitos da intervenção se generalizaram ao longo do tempo. Apesar disso, de modo geral, todos os participantes passaram a consumir maior variedade de alimentos de um mesmo grupo alimentar quando se compara a fase de linha de base com a fase de intervenção. Pesquisas futuras poderiam controlar melhor os alimentos servidos nas diferentes fases do estudo, bem como sua forma de preparo (in natura, como no caso das frutas, ou preparados, como 
no caso da lasanha, por exemplo), já que dados de outra pesquisa sugerem que o fato de os participantes não saberem como o alimento é preparado aumenta a probabilidade de que sua escolha seja feita antes da refeição (Brandina, Panosso, \& Souza, 2016).

Outro aspecto a ser observado é o fato de que, nas cartas do bruxo, havia a presença de instruções com autoclíticos (e.g., "No almoço tinha arroz, batata palha, feijão, alface e carne e você só comeu alface e batata palha. Escolher só dois alimentos para comer não é bom para a saúde. Perca 1 ponto"). O autoclítico é um comportamento verbal de segunda ordem sob controle do comportamento verbal do próprio falante e influencia o ouvinte em uma direção específica. O autoclítico comenta ou qualifica outras partes desse comportamento, ao descrever sua força, apontar as circunstâncias que o controlam, negar informações posteriores etc. (Skinner, 1978). Por exemplo, a sentença "...Escolher só dois alimentos para comer não é bom para a saúde" pode ter qualificado o comportamento de escolher apenas dois tipos de alimentos como prejudiciais e favorecido a emissão de respostas dos participantes de selecionar maior variedade de alimentos, tanto no jogo quanto nas refeições. Uma pesquisa conduzida por Baptistussi (2010) mostrou que instruções com autoclíticos produziram mudança na escolha de alimentos entre diferentes grupos alimentares.

Por fim, no jogo Cestinha Mágica, comportamentos verbais relativos aos comportamentos da classe de selecionar alimentos variados eram seguidos por reforçadores específicos (pontos, elogios). O reforço dos comportamentos verbais relacionados a essa classe pode produzir mudanças não apenas nos comportamentos verbais dos jogadores como também nos não-verbais (Catania, Matthews, \& Shimoff, 1982; Dias \& Hübner, 2003). Dessa forma, os comportamentos aprendidos nesse contexto podem generalizar-se para outros (e.g., hora da refeição) e, caso sejam emitidos, eles podem ser reforçados pelo sabor dos alimentos ou elogios dos adultos presentes e, por conseguinte, serem fortalecidos.

Apesar dos resultados obtidos, quando se utilizam jogos para a coleta de dados, uma das limitações diz respeito às muitas variáveis presentes como, por exemplo, a interação entre os jogadores e as variáveis arranjadas por meio do jogo para o ensino de habilidades específicas. No jogo Cestinha Mágica, a variedade de casas que o compõem e suas diferentes funções, além de contribuírem para torná-lo motivador, levantam questões acerca da interferência das instruções, do uso de estímulos reforçadores específicos e do uso de operantes verbais autoclíticos sobre as respostas de consumo alimentar. Pesquisas futuras poderão investigar os efeitos de cada uma dessas variáveis sobre a seleção de alimentos para o consumo.

Embora preferissem determinados alimentos, os participantes desse estudo não apresentavam restrições alimentares severas. O P3, por exemplo, consumiu todas as verduras, legumes e frutas que foram oferecidos, deixando de consumir apenas alguns tipos de alimentos do grupo dos cereais/grãos e das proteínas. Pesquisas futuras poderiam ter como participantes crianças que fizessem escolhas alimentares mais restritivas que as crianças deste estudo, o que possibilitaria avaliar, de forma mais incisiva, os efeitos do jogo sobre as escolhas e o consumo de alimentos dos diferentes grupos. Ressalta-se que a aceitação de alimentos ocorre em razão da exposição repetida a eles (Silva, Pais-Ribeiro, \& Cardoso, 2008); é, porém, necessário que experimentem o alimento novo, mesmo que em quantidade mínima, para que sua aceitação aumente (Ramos \& Stein, 2000).

Por fim, modelar o comportamento alimentar da criança exige atenção para uma série de aspectos tais como: ao introduzirem-se novos alimentos, faz-se necessário reapresentar 
o alimento diversas vezes e de diferentes formas; apresentar os alimentos separadamente para a criança conhecê-los e para habituar seu paladar aos novos sabores; estar atento às contingências que se estabelecem no momento das refeições e quais são as contingências estabelecidas ao se oferecerem alimentos não saudáveis às crianças; prestar atenção às regras estabelecidas para a refeição; qual o modelo de consumo os pais estão dando aos fiIhos ao consumirem certos alimentos, e aprender a identificar a diferença entre saciedade e fome (Heller, 2004).

O jogo pode ser uma tecnologia capaz de proporcionar, de forma reforçadora, o contato da criança com os alimentos; os pais são fundamentais para que a manutenção do comportamento de um consumo alimentar variado ocorra no dia a dia. Futuras pesquisas poderiam verificar os efeitos do uso do jogo sobre o consumo alimentar de crianças quando elas jogam com os pais. Por fim, o jogo trabalha com os três grupos alimentares, indicados para o consumo diário: verduras/legumes/frutas, cereais/grãos e proteínas/laticínios e a atribuição de pontos aos grupos alimentares seguiu as recomendações do Dietary Guidelines e MyPlate. Pesquisas futuras poderiam acrescentar outros tipos de alimentos aos apresentados pelo jogo, readequá-los aos grupos alimentares sugeridos pela ciência da nutrição e definir a pontuação seguindo os padrões nutricionais de referência da população brasileira.

O jogo, associado a outras intervenções e técnicas terapêuticas, pode ser uma estratégia capaz de proporcionar um contexto de aprendizagem relacionado à educação alimentar de forma divertida e motivadora. As escolhas alimentares da idade adulta estão relacionadas àquelas aprendidas na infância; assim, é de fundamental importância o desenvolvimento de tecnologias e intervenções que auxiliem a criança a fazer escolhas e, consequentemente, ter comportamentos alimentares mais saudáveis.

\section{Referências}

Abreu, P. R., \& Hübner, M. M. C. (2011). Efeitos de instruções sobre respostas de checagem. Psicologia: Teoria e Pesquisa, 27, 301-308.

Albuquerque, L. C., \& Paracampo, C. C. P. (2010). Análise do controle por regras. Psicologia USP, 21(2), 253-273.

Baptistussi, M. C. (2010). O efeito de variáveis verbais e não verbais sobre o comportamento de escolha de alimentos em crianças. (Tese de Doutorado, Programa de Pós-Graduação em Psicologia Experimental, Universidade de São Paulo, São Paulo). Disponível em http:// www.teses.usp.br/teses/disponiveis/47/47132/tde-09022011-103124/pt-br.php

Birch, L. L. (1992). Children's preferences for high-fat foods. Nutrition reviews, 50(9), 249255. doi: 10.1111/j.1753-4887.1992.tb01341.x

Birch, L. L. (1999). Development of food preferences. Annual Reviews, 19, 41-62. doi: 10.1146/annurev.nutr.19.1.41

Birch, L. L., McPhee, L., Shoba, B. C., Pirok, E., \& Steinberg, L. (1987). What kind of exposure reduces children's food neophobia? Looking vs. tasting. Appetite, 9(3), 171-178.doi: 10.1016/S0195-6663(87)80011-9

Brandina, M. C. G., Panosso, M., \& Souza, S. R. (2016). O uso de um jogo de tabuleiro educativo sobre a variação da escolha de alimentos: papel do autoclítico e da forma de apresentação 
dos alimentos. In J. C. Luzia, G. B Filgueiras, A. E. Gallo, \& J. Gamba (Eds.), Psicologia e análise do comportamento: Saúde, educação e processos básicos (pp. 73-88). Londrina, PR: Universidade Estadual de Londrina. Disponível em http://www.uel.br/pos/pgac/ wp-content/uploads/2017/03/PSICOLOGIA-E-AN\%C3\%81LISE-DO-COMPORTAMENTOSA\%C3\%9ADE-EDUCA\%C3\%87\%C3\%83O-E-PROCESSOS-B\%C3\%81SICOS.pdf

Brasil, A. L. D., Devncenzi, M. U., Ribeiro, L. C. (2007). Nutrição infantil. In S. M. C. Silva, \& J. D. P. Mura. Tratado de alimentação, nutrição \& dietoterapia. São Paulo: Rocca.

Cardoso, J. B. (2007). Escolha de acordo com o modelo: Relações emergentes entre estímulos arbitrários em indivíduos de diferentes condições de peso corpóreo (Dissertação de Mestrado, Programa de Estudos Pós-Graduados em Psicologia Experimental, Pontifícia Universidade Católica de São Paulo, São Paulo). Disponível em https://sapientia.pucsp.br/ handle/handle/16802

Catania, A. C., Matthews, B. A., \& Shimoff, E. (1982). Instructed versus shaped human verbal behavior: Interactions with nonverbal responding. Journal of the Experimental Analysis of behavior, 38(3), 233-248. doi: 10.1901/jeab.1982.38-233

Costa, M.R. (2016). A influência da mídia televisiva nas escolhas alimentares das crianças e na obesidade infantil: uma revisão da literatura. Faculdade de Ciências da Educação e da Saúde. (Trabalho de Conclusão de Curso, Curso Nutrição, UniCEUB). Disponível em http:// repositorio.uniceub.br/bitstream/235/11200/1/TCC\%20Midi\%C3\%A3\%20Ribeiro.pdf

Dias, F. C., \& Hübner, M. M. C. (2003). Comportamento verbal e não verbal: efeitos do reforçamento de tactos com autoclíticos referentes ao ler sobre o tempo despendido com leitura. In H. M. Sadi \& N. M. S. Castro (Eds.), Ciência e comportamento humano: Conhecer e avançar (pp. 163-173, v. 3). Santo André, SP: ESETec.

Dietary Guidelines e MyPlate. Disponível em https://health.gov/dietaryguidelines/2015/resources/2015-2020_Dietary_Guidelines.pdf

Gris, G., Alves, H. W., Assis, G. J. A., \& Souza, S. R. (2017). Utilização de jogos adaptados para avaliação de habilidades matemáticas e monetárias. Temas em Psicologia, 25(3), 11391152. doi: 10.9788/TP2017.3-12Pt

Heller, D. C. L., Souza, D. P., Scur, E.M.A., Pinto, M. S., \& Castilho, S. (2004). Obesidade infantil. Manual de prevenção e tratamento. Santo André, SP: ESETec.

Hübner, M. M. C., Austin, J., \& Miguel, C. F. (2008). The effects of praising qualifying autoclitics on the frequency of reading. The Analysis of verbal behavior, 24(1), 55-62. doi: 10.1007/BF03393056

Lowe, C. F., \& Horne, P. J. (2009). Food dudes: Increasing children's fruit and vegetable consumption. Cases in Public Health Communication \& Marketing, 3, 161-185.

Michael, J. (1993). Establishing operations. The Behavior Analyst, 16(2), 191-206. doi: 10.1007/BF03392623

Ministério da Saúde do Governo Brasileiro. Portal da saúde. (2006). Guia Alimentar para a População Brasileira. Disponível em http://www.saude.gov.br

Oliveira, J. A. N, Araújo, A. M. M, Soares, C. S., \& Coimbra, J. R. (2016). A influência da família na alimentação complementar: Relato de experiências. DEMETRA: Food, Nutrition and Health, 11(1), 75-90. doi: 10.12957/demetra.2016.16053

Panosso, M. G., \& Souza, S.R. (2014). Equivalência de estímulos: Efeitos de um jogo de tabuleiro sobre escolhas alimentares. Acta Comportamentalia, 22(3), 315-333. 
Ramos, M., \& Stein, L. M. (2000). Desenvolvimento do comportamento alimentar infantil. Jornal de Pediatria, 76, 229-237.

Rossi, A., Moreira, E. A. M.,\& Rauen, M. S. (2008). Determinantes do comportamento alimentar: uma revisão com enfoque na família. Revista de Nutrição, 21(6), 739-748.

Santos, S. L. (2017). Influência de personagens infantis sobre escolhas alimentares em crianças: Um estudo com equivalência de estímulos (Tese de Doutorado, Programa de PósGraduação em Psicologia, Universidade Federal de São Carlos, São Carlos). Disponível em https://repositorio.ufscar.br/handle/ufscar/9023

Sidman, M. (1971). Reading and auditory-visual equivalences. Journal of speech and Hearing Research, 14(1), 5-13. doi:10.1044/jshr.1401.05

Sidman, M., \& Tailby, W. (1982). Conditional discrimination vs. matching to sample: An expansion of the testing paradigm. Journal of the Experimental Analysis of Behavior, 37(1), 5-22. doi: 10.1901/jeab.1982.37-5

Silva, I., Pais-Ribeiro, J. L., \& Cardoso, H. (2008). Porque comemos o que comemos: Determinantes psicossociais da selecção alimentar. Psicologia, Saúde \& Doenças, 9(2), 189-208.

Siqueira, E. S., Barros, S. E., Marques, B. L., \& Monteiro, C. D (2011). Um jogo com reconhecedor de voz para o ensino de crianças com dificuldade de aprendizagem em leitura e escrita. Anais do XXXI Congresso da Sociedade Brasileira de Computação (CSBC). Disponível em http://www.dimap.ufrn.br/csbc2011/anais/eventos/contents/CADERNO.pdf

Skinner, B. F. (1978). O comportamento verbal (M. P. Villalobos, Trad.). São Paulo: Cultrix. (Obra original publicada em 1957).

Smeets, P. M., \& Barnes-Holmes, D. (2003). Children's emergent preferences for soft drinks: Stimulus-equivalence and transfer. Journal of Economic Psychology, 24(5), 603-618. doi: 10.1016/S0167-4870(03)00004-7

Steele, D., \& Hayes, S. C. (1991). Stimulus equivalence and arbitrarily applicable relational responding. Journal of the Experimental Analysis of Behavior, 56(3), 519-555. doi: 10.1901/ jeab.1991.56-519

Straatmann, G. (2008). A utilização do paradigma de equivalência de estímulos para modificar a preferência alimentar (Dissertação de Mestrado, Programa de Pós-Graduação em Psicobiologia, Universidade de São Paulo, Ribeirão Preto). Disponível em www.teses.usp. br/teses/disponiveis/59/59134/tde-12052009-141159/publico/STRAATMANN.pdf

Straatmann, G., Almeida, S. S., \& de Rose, J. C. (2014). Computerized assessment of food preferences in adolescents in the stimulus equivalence paradigm. Temas em Psicologia, 22(3), 613-624. doi: 10.9788/TP2014.3-07

Teixeria, V. H., \& Moreira, P. (2016). Maternal food intake and socioeconomic status to tackle childhood malnutrition. Jornal de Pediatria, 92(6),546-548.

Viana, R. C., \& Banaco, R. A. (2010). Obesidade e análise do comportamento alimentar: Revisão e novos paradigmas de pesquisa. In G. Couto \& S. D. Pires (Eds.), Contornos da Psicologia Contemporânea (pp. 105-142). Itatiba, SP: Casa do Psicólogo.

Wardle, J., \& Cooke, L. (2008). Genetic and environmental determinants of children's food preferences. British Journal of Nutrition, 99(1), 15-21. doi: 10.1017/S000711450889246X. World Health Organization. (2017). New global estimates of child and adolescent obesity released on World Obesity Day. Disponível em http://who.int/end-childhood-obesity/news/ 
new-estimate-child-adolescent-obesity/en/

Xander, P., Haydu, V. B., \& Souza, S. R. (2016). "Dim Dim: negociando e brincando" no ensino de habilidades monetárias a pré-escolares. Revista CES Psicología, 9(1), 65-88.

Recebido: $27 / 07 / 2017$

Última revisão: 15/12/2017

Aceite final: 30/01/2018

\section{Sobre os autores:}

Mariana Gomide Panosso: Doutoranda do Programa de Pós-Graduação em Psicologia da Universidade Federal de São Carlos. Mestre em Análise do Comportamento pela Universidade Estadual de Londrina. Docente do Grupo Rhema Educação e Psicóloga clínica. E-mail: mariana_panosso@hotmail.com

Gabriele Gris: Doutoranda do Programa de Pós-Graduação em Psicologia da Universidade Federal de São Carlos. Mestre em Análise do Comportamento pela Universidade Estadual de Londrina. E-mail: grisgabriele@gmail.com

Silvia Regina de Souza: Doutora em Psicologia Clínica pela Universidade de São Paulo; Pós-doutorado em Motricidade Humana pela Universidade Técnica de Lisboa e em Psicologia Experimental pela Universidade de São Paulo. Professora associada do Departamento de Psicologia Geral e Análise do Comportamento e do Programa de Mestrado em Análise do Comportamento da Universidade Estadual de Londrina. Ex-coordenadora do Programa de Mestrado em Análise do Comportamento da Universidade Estadual de Londrina (gestão 2013-2016). Coordena o LADEJE (Laboratório de Desenvolvimento e Avaliação de Jogos Educativos).E-mail: ssouza.arrabal@gmail.com 
